\title{
Therapeutischer Nutzen
}

Die therapeutische Nutzbarkeit von Sauerstoff als Medikament ist sowohl dem medizinischen Fachpersonal als auch der Allgemeinbevölkerung oft nur spärlich bekannt. Mit der hyperbaren Oxygenation steht der modernen Medizin jedoch ein Verfahren zur Verfügung, Sauerstoff für eine Reihe von Erkrankungen therapeutisch einzusetzen. Auf der Basis der drei physikalischen Gasgesetze geht unter hyperbaren Bedingungen ein Vielfaches an Sauerstoff im Blutplasma in Lösung über, als dies unter normalem Umgebungsdruck der Fall ist. Für den menschlichen Organismus resultiert daraus eine Reihe von biochemischen und physikalischen Veränderungen auf Blutzellen, Gefäße, Bakterien, Knochen- und Weichteilgewebe. Untrennbar mit der $\mathrm{HBO}$ verbunden ist das Fachgebiet der Tauchmedizin durch die Anwendung der HBO bei Dekompressionserkrankung und Gasembolie.

D. Maurer, Hyperbare Oxygenation und Tauchmedizin, essentials, DOI 10.1007/978-3-658-11713-9_1 